\title{
A spatial explanation for synchrony biases in perceptual grouping: Consequences for the temporal-binding hypothesis
}

\author{
GUY WALLIS \\ Max Planck Institute for Biological Cybernetics, Tübingen, Germany \\ and University of Queensland, Brisbane, Queensland, Australia
}

\begin{abstract}
If two images are shown in rapid sequential order, they are perceived as a single, fused image. Despite this, recent studies have revealed that fundamental perceptual processes are influenced by extremely brief temporal offsets in stimulus presentation. Some researchers have suggested that this is due to the action of a cortical temporal-binding mechanism, which would serve to keep multiple mental representations of one object distinct from those of other objects. There is now gathering evidence that these studies should be reassessed. This article describes evidence for sensitivity to fixational eye and head movements, which provides a purely spatial explanation for the earlier results. Taken in conjunction with other studies, the work serves to undermine the current body of behavioral evidence for a temporal-binding mechanism.
\end{abstract}

The human brain contains many functionally discrete regions involved in the processing of specific object attributes, such as form, color, motion, and location (Felleman \& Van Essen, 1991; Ungerleider \& Haxby, 1994; Zeki et al., 1991). A current issue in visual perception is understanding the mechanism by which these disparate representations remain in correspondence with one another and yet distinct from the attributes of other, simultaneously perceived objects (Reynolds \& Desimone, 1999). A popular explanation is that all the neurons responsive to a particular object would temporally synchronize their firing across the visual cortex to bind the various representations (Malsburg, 1995; Singer \& Gray, 1995). This proposal has received backing from several neurophysiological studies, although the results remain controversial (Singer \& Gray, 1995; Tovee \& Rolls, 1992). In addition to the neurophysiological work, several behavioral studies have described how perceptual grouping emerges from (Lee \& Blake, 2001) or is influenced by (Leonards, Singer, \& Fahle, 1996; Parton, Donnelly, \& Usher, 2001; Usher \& Donnelly, 1998), the temporal synchrony of stimulus presentation. Some authors have been guarded

This work was supported by the Max Planck Society, Germany, and the Australian Research Council. I am grateful to Marius Usher, Allison Sekuler, and two anonymous referees for insightful and constructive criticism of an earlier draft of this article. I am also grateful to Andre Camilleri and Craig Mattocks for their assistance in running the experiments, to Per Magne Knutsen for his literature review on fixational eye movements, and to Adam Reeves, Heinrich Bülthoff, Mike Land, Ulrike Siebeck, Rob McIntosh, Heiner Deubel, and James Tresilian for useful discussion and comments on the experiments. Correspondence concerning this article should be addressed to G. Wallis, Perception and Motor Systems Laboratory, Connell Building, University of Queensland, QLD 4072 Australia. in their postulation of mechanisms that could underlie such sensitivity (Lee \& Blake, 2001), whereas others see it as evidence for the time-based binding mechanism described above (Leonards et al., 1996; Parton et al., 2001; Usher \& Donnelly, 1998).

The behavioral studies have themselves generated a great deal of correspondence and controversy over the last few years, as has been detailed in a succinct review by Farid (2002). Early reports of temporal sensitivity by Fahle (1993), for example, were later attributed to spurious motion cues (Morgan \& Castet, 2001). Indeed, as Fahle himself described, some subjects reported perceiving apparent motion in the displays, consistent with the earlier work of Westheimer and McKee (1977), who attributed their temporal hyperacuity effects to motionprocessing mechanisms. Later work by Lee and Blake (1999b), aimed at circumventing these types of motion cue, has itself been criticized on the basis of contrast and motion artifacts (Adelson \& Farid, 1999; Farid \& Adelson, 2001; Lee, \& Blake, 1999a; Morgan \& Castet, 2001), and subsequent results from the same group (Lee \& Blake, 2001) have also produced controversy (Farid, 2002). In more recent work, Fahle and colleagues have once again attempted to counter earlier criticisms (Kandil $\&$ Fahle, 2001), but they have reported effects that were, at times, an order of magnitude too long to be of direct relevance to a temporal-binding mechanism (Farid, 2002; Morgan \& Castet, 2001). Other recent studies have offered alternative criticisms. Beaudot (2002), for example, suggested that in the case of contour detection, the order of presentation (contour/background) might play a role in a subject's sensitivity to stimulus asynchrony. Dakin and Bex (2002) reported that altering stimulus contrast, or the use of a mask, reduces or even abolishes 
the effectiveness of temporal offset in the same task. Interestingly, they actually failed to replicate the results of Beaudot in their work.

Within all of this controversy, there is one factor that has received relatively little close attention, the relevance of which becomes apparent only when one considers the format of many of these experiments. Many of the studies have focused on the use of a grid of elements. By altering the precise moment at which the elements are displayed, particular interpretations can be instantiated or at least promoted-be it rows rather than columns (Parton et al., 2001; Usher \& Donnelly, 1998), specific patches of adjacent elements (Leonards et al., 1996), or curved "paths" of oriented elements (Lee \& Blake, 2001). The perceptual grouping of elements within a grid has been studied for many years and was a favorite topic of the early Gestalt psychologists. In his classic treatise on the subject, Wertheimer (1923) presents example configurations of such grids, describing how observer preferences can be biased by making minor alterations to the arrangement of the grid elements. For instance, subjects tend to report seeing rows, rather than columns, if the elements are arranged more closely horizontally (proximity rule) or if the linearity of the columns is disrupted (good continuation). Equal and opposite results are obtained if the arrangement of the elements is reversed. Sensitivity to these types of cues opens an alternative avenue for biasing the perception of temporally asynchronous stimulinamely, image shifts caused by eye and head movements. Any image motion from one frame to the next will result in a spatial shift in the location of the grid elements as they appear on the retina. Any such shift will almost certainly result in the disruption of the row and column arrangement of the elements and, hence, will affect observer responses.

Even at the short presentation times typical of the behavioral studies listed above (10-20 msec), a saccadic eye movement would result in a large shift of grid elements from one image frame to the next. Consistent with this idea, Dakin and Bex (2002) reported a significant decrease in sensitivity to temporal offsets when using retinally stabilized images. Aware of such problems, researchers in the field invariably require their observers to fixate both before and during stimulus presentation, citing this as a reason that eye movement artifacts can be discounted (Fahle, 1993; Usher \& Donnelly, 1998). Fahle actually used an eye tracker to discount trials in which the point of fixation changed. Unfortunately, even if one can assume that fixation is maintained, there remain several sources of eye movement, not the least of which are involuntary eye movements (Adler \& Fliegelman, 1934; Carpenter, 1988; Ratliff \& Riggs, 1950). Despite their small amplitude and the fact that they are not directly perceptible, fixational eye movements have been implicated in perceptual phenomena such as the jitter aftereffect (Murakami \& Cavanagh, 1998) and Leviant's enigma (Mon-Williams \& Wann, 1996). The three major cate- gories of fixational eye movement are drift, microsaccades, and tremor (Adler \& Fliegelman, 1934; Carpenter, 1988; Ratliff \& Riggs, 1950). Although drift results in reasonably large amplitude movements, its speed is relatively low (Ratliff \& Riggs, 1950), and so it would not result in a large stimulus shift from one frame to the next. Microsaccades, on the other hand, can be both large and fast, and so might play a role, although their relative infrequency (approximately one every $500 \mathrm{msec}$, according to Coakley, 1983) would limit their influence over many trials. The third form of eye movement, ocular microtremor (OMT, or physiological nystagmus), has a relatively small amplitude but is both fast and continuous (Bolger, Bojanic, Sheahan, Coakley, \& Malone, 1999; Carpenter, 1988; Ratliff \& Riggs, 1950), making it a strong candidate for producing eye movement influence in rapid presentation paradigms. The maximum offsets one would expect in a pair of rows of elements shown for two consecutive 10-msec intervals are shown in Table 1, with derivation of the OMT values described in the Appendix. Clearly, the offsets are very small. Nonetheless, humans are extremely sensitive to the collinearity and proximity of grid elements (Ben-Av \& Sagi, 1995; Fahle $\&$ Koch, 1995), and so even very small offsets have the potential to affect subject responses. What is more, in the absence of bite-bars or similar levels of head restraint, such effects become even larger, due to natural fixational head movements (Ferman, Collewijn, Jansen, \& Van den Berg, 1987).

Overall, the work of Dakin and Bex (2002), Farid and Adelson (2001), Morgan and Castet (2001), and others provide credible reasons (such as the asymmetric effects of sudden stimulus onset, motion and contrast artifacts, and saccadic eye movements) for doubting the role of temporal sensitivity in contour detection. The purpose of this study is to describe in detail the role of fixational eye movements as another potentially confounding cue, one that has relevance to a wide range of reports of the temporal characteristics of stimuli causing or affecting perceptual grouping.

Table 1

Characteristics of the Three Types of Involuntary Eye Movement, Along With the Maximum Displacement of the Eye Experienced Between Frames of a 100-Hz Computer Monitor Display Similar to that Used in Experiments 2 and 3

\begin{tabular}{llll}
\hline & $\begin{array}{c}\text { Amplitude } \\
\text { (deg) }\end{array}$ & \multicolumn{1}{c}{ Motion } & $\begin{array}{c}\text { Maximum } \\
\text { Displacement } \\
\Psi(\mathrm{deg})\end{array}$ \\
\hline Drift & 0.07 & linear $0.017 \mathrm{deg} / \mathrm{sec}$ & 0.0002 \\
Tremor & 0.008 & cyclic $50-100 \mathrm{~Hz}$ & 0.011 \\
Microsaccades & 0.07 & linear $4.7 \mathrm{deg} / \mathrm{sec}$ & 0.047 \\
\hline
\end{tabular}

Note-The translation component in this period due to oculomotor microtremors (OMTs) and microsaccades is much larger than that due to drift. The figures are only approximate, since reports differ in the literature as to the precise values and will depend on the particular individual (Bolger, Bojanic, Sheahan, Coakley, \& Malone, 1999; Carpenter, 1988; Ratliff \& Riggs, 1950). More information for the process of calculating the amplitude of OMT influence is given in the Appendix. 


\section{EXPERIMENT 1 Baseline Sensitivity to Spatial Offsets}

The purpose of this first experiment was to establish whether or not a subject's behavior could possibly be influenced by the tiny offsets associated with OMT. To test this, a standard synchronous grid stimulus was employed. The only difference from a standard, regular grid was the introduction of subtle spatial offsets of the elements, of the type and magnitude that theory predicts OMT would produce. By presenting the stimuli wholly synchronously with known spatial offsets, the stimulus simulated the spatial offset associated with OMT in an asynchronous presentation, while circumventing the actual influence of eye movements on performance.

\section{Experiment 1A}

\section{Method}

During the experiment, the subjects viewed a stimulus grid consisting of $8 \times 8$ circular elements. The stimuli were presented on a Sony Trinitron CRT monitor (P22 phosphors) at a refresh rate of $96 \mathrm{~Hz}$. Images were presented using a Silicon Graphics Onyx300 running an Infinite Reality II graphics engine, which uses $8 \times 8$ pixel multisampling to produce a fully antialiased 1,280 $\times 1,024$ pixel display. Each trial was initiated by the observer via a buttonpress, after which a fixation cross appeared in the center of the screen for $1.5 \mathrm{sec}$, followed by the stimulus. Head placement was regulated by means of a combined chin and forehead rest. The subjects viewed the stimuli at a range of $600 \mathrm{~mm}$, at which point the grid subtended an angle of $5^{\circ}$. During an individual trial, all of the grid elements appeared simultaneously for a single frame $(\approx 10 \mathrm{msec})$. This is referred to henceforth as the SYNC condition (see Figures 1A and B). On half of the trials, alternate rows of elements were offset in a random direction, and in the other half, alternate columns were offset.
Ten subjects took part in the experiment, and their task was simply to indicate, via a buttonpress, whether they perceived the grid of elements as being organized in rows or columns. In this experiment and all the subsequent ones, the subjects had corrected-to-normal vision, were in the age range of 21-40 years, and unless indicated, were naive as to the purpose of the experiment. The stimuli were presented as red dots on a dull gray background in a darkened room. Red was chosen because persistence of the P22 red channel is minimal, with most of the stored energy dissipating within $2 \mathrm{msec}$. This is not true of the green and blue channels, which despite decaying quickly initially, produce a visible afterglow for a much longer period (Kuhn, 2002; Sherr, 1993). Mean screen luminance was $11 \mathrm{~cd} / \mathrm{m}^{2}$, with a stimulus Michelson contrast of $20 \%$.

\section{Results}

The binary nature of the row/column decision lends itself to the use of signal detection theory (Green \& Swets, 1974). In all three experiments, observer $d^{\prime}$ values were calculated on the basis of the correspondence between their actual and the predicted responses, given the type of spatial offset. Figure 2A displays the results of the first experiment. As one would expect, at $0^{\circ}$ offset, the subjects were responding at chance level (corresponding to a $d^{\prime}$ of zero). However, for any spatial offset above $0^{\circ}$, the observers demonstrated a consistent and significant sensitivity to the offset. In fact, performance for offsets of as little as $0.01^{\circ}$ were significantly above chance $[t(9)=3.351, p<.01]$. Referring back to Table 1 , we see that eye movements could produce spatial shifts in the retinal image of this size and greater. Hence, the first experiment demonstrated that subjects are sensitive to spatial offsets of sufficiently small amplitude that OMT eye movements could potentially explain earlier reports of sensitivity to temporal asynchrony.
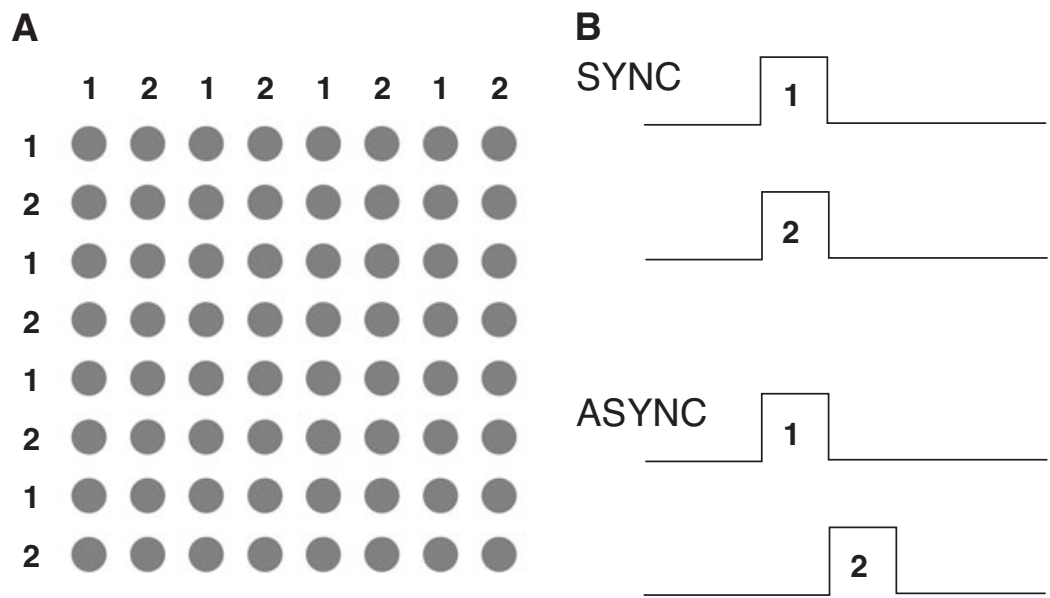

Figure 1. (A) The stimulus grid. In Experiments 1A and 1B, alternate rows or columns (labeled 1 and 2 in the figure) were presented synchronously (SYNC) and spatially offset in a random direction, with one of five possible amplitudes. In Experiments 2 and 3, alternate rows or columns were not offset spatially but, rather, temporally (ASYNC). (B) Summary of the SYNC and ASYNC presentation paradigms. In the SYNC case, all the dots appeared simultaneously. In the ASYNC case, those rows (columns) labeled 1 above appeared simultaneously for a fixed period and then disappeared, followed immediately by the alternate rows (columns), labeled 2 , for an equivalent period. 
A

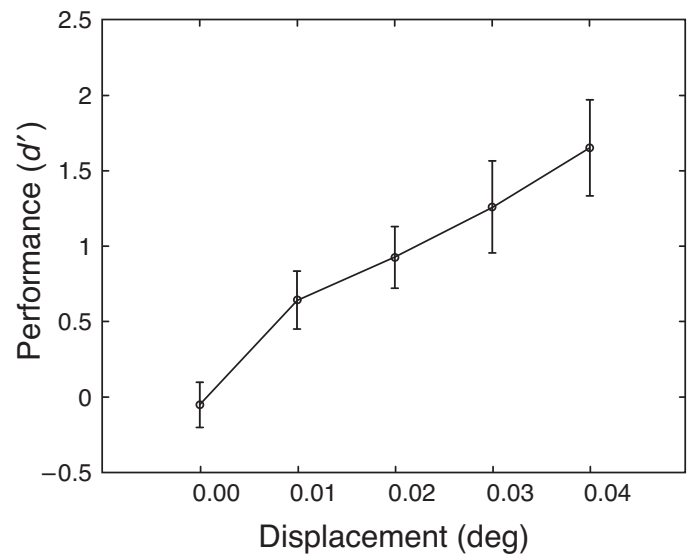

B

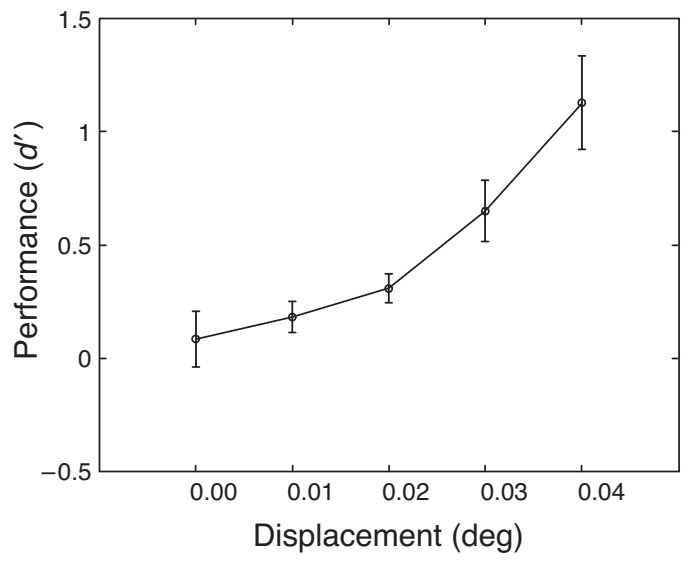

Figure 2. (A) Results from Experiment 1A, revealing a steady increase in performance as the amplitude of the spatial offset increases. As one would expect, the subjects showed chance performance at zero offset but were performing well above chance even for offsets as small as one hundredth of a degree, revealing significant levels of sensitivity to an even minor displacement of the elements. (B) Results from Experiment 1B, which recreated the conditions used in an earlier study (Usher \& Donnelly, 1998) with synchronous presentation of spatially offset stimuli. Performance was above chance for even the smallest offset of $0.01^{\circ}$, demonstrating that the minor spatial offsets caused by oculomotor tremors can account for the performance measured in that study. Error bars represent the standard error of the mean performance across subjects in both graphs.

\section{Experiment 1B}

\section{Method}

The first experiment was also run under the conditions used in an earlier report of sensitivity to temporal asynchrony made by Usher and Donnelly (1998). Their experimental paradigm was very similar to that adopted in Experiment 1A. The major differences were the precise presentation time and the number of elements in the grid. Their experiment proceeded in the same manner as that in Experiment $1 \mathrm{~A}$ described above, except that their frame duration was $15 \mathrm{msec}$ and they used 36 elements arranged in a $6 \times 6$ grid. In order to reproduce these conditions as closely as possible, in this follow-up experiment, a refresh rate of $60 \mathrm{~Hz}$ was used, producing a frame duration of approximately $16.7 \mathrm{msec}$. All the elements of the grid were displayed synchronously for a single frame with alternate rows (or columns) slightly spatially offset from one another. Viewing distance was set at $1,570 \mathrm{~mm}$, so that a single pixel subtended exactly $0.01^{\circ} \times 0.01^{\circ}$ of visual angle. The grid was enlarged in order that it once again subtended an angle of 5.0 . Six new subjects took part in this version of the experiment.

\section{Results}

The results for this experiment appear in Figure 2B. As in Experiment 1A, at zero amplitude offset, the observers were performing at chance level. However, once again, even at an offset amplitude of $0.01^{\circ}$, sensitivity rose above chance $[t(5)=2.66, p<.05]$. When an asynchronous version of the grid is viewed, one might well expect eye movements to result in retinal image shifts of this size. By way of comparison, for the shortest presentation period used in their experiments, Usher and Donnelly (1998) reported performances of around 68\% for low-contrast and $85 \%$ for high-contrast stimuli. This level of performance was achieved here only for spatial offsets on the order of $0.03^{\circ}$ and $0.04^{\circ}$, which produced performance levels of $63 \%$ and $70 \%$, respectively. One possible explanation for this is that despite the use of a chinrest in these experiments, the head also moved during fixation. As was mentioned earlier, the likely effect of this is actually to as much as double the velocity and amplitude of retinal image motion (Ferman et al., 1987) and, hence, to increase the size of induced spatial shifts in the asynchronously displayed image. A prediction of this study is that image shifts should be on the order of $0.03^{\circ}$ - a prediction that was tested in Experiment 3. Ultimately, it appears that subjects are sufficiently sensitive to minor spatial offsets that their performance in the asynchronous case described by Usher and Donnelly could be attributed to a combination of lateral (head movement induced) and rotational (eye rotation induced) eye movements.

\section{EXPERIMENT 2 Effects of Viewing Distance}

Experiment 2 was an attempt to establish a role for eye movements by testing the effect of viewing distance on performance. The simple concept behind this was that if eye movements are producing a spatial shift in the pattern to which subjects are sensitive, by having them sit farther away from the stimulus any such effects should increase, since the effective retinal shift produced by an eye movement of fixed angular amplitude will be correspondingly larger. This assumes, of course, that subjects are sensitive to the degree of displacement of the grid elements in proportion to the grid itself, and not to visual angle alone.

\section{Method}

The observers were shown a grid of elements in which alternate rows (or columns) appeared for $10 \mathrm{msec}$, followed by the neigh- 
boring rows (or columns; ASYNC condition; see Figures 1A and 1B) - in general accord with the temporal offset experiments of various previous studies (Lee \& Blake, 2001; Leonards et al., 1996; Parton et al., 2001; Usher \& Donnelly, 1998). In addition to the ASYNC condition, a further SYNC condition of the form used in Experiments 1A and 1B was run. The purpose of including this condition was to see whether any changes in performance in the ASYNC condition would be mirrored by a stimulus containing a purely spatial offset. The magnitude of this offset $\left( \pm 0.01^{\circ}\right)$ was chosen to match the spatial shift predicted to be caused by eye movements in the ASYNC condition. The $8 \times 8$ grid of Experiment $1 \mathrm{~A}$ was used, and performance was tested at four viewing distances: $0.5,1,2$, and $4 \mathrm{~m}$ (see Figure $3 \mathrm{~A}$ ). The 10 subjects who participated in Experiment 1A took part in this experiment as well. The grid subtended an angle of $10^{\circ}$ at $500 \mathrm{~mm}$.

\section{Results}

The results of Experiment 2 appear in Figure 3B. Irrespective of viewing distance, the subjects demonstrated a clear sensitivity to presentation asynchrony, consistent with earlier reports. However, results also reveal an increased sensitivity as viewing distance increases, consistent with the eye movement hypothesis. The figure also shows performance for synchronized presentation with a spatial offset of $0.02^{\circ}$. The immediate impression is that the two graphs are remarkably similar in both form and magnitude. Effect sizes were measured via linear regression analysis of individual subject performance versus the log of the viewing distance. Across all subjects, for the ASYNC condition $.86 \leq r^{2} \leq .999$ with a mean $r^{2}$ of .95 , and for the SYNC condition $.924 \leq r^{2} \leq .996$ with a mean $r^{2}$ of .96 , revealing good correspondence to a linear trend in loglinear coordinates in both cases. An analysis of variance of the gradients of the fitted lines revealed no significant difference between the two conditions $\left[F(1,9)=1.123, M S_{\mathrm{e}}=0.0304\right.$, n.s. $]$. Hence, it

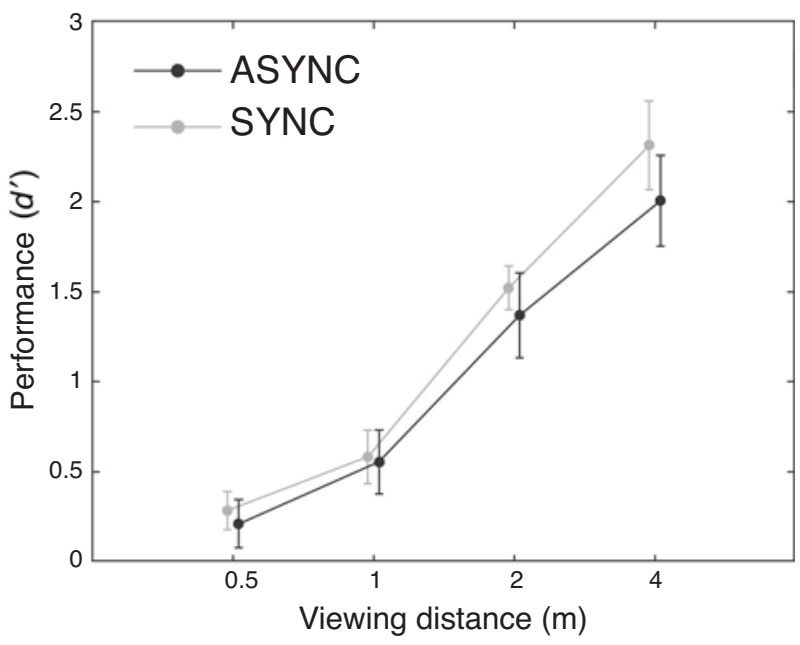

Figure 3. (A) Results from Experiment 2, in which alternate rows or columns appeared asynchronously. As is predicted by an eye-movement-based explanation of sensitivity to asynchronous presentations, the greater the viewing distance, the better performance becomes. Error bars represent the standard error of the mean performance across subjects. appears that the effect of altering viewing distance in the ASYNC condition can be matched by making spatial offsets of fixed visual angle, in the order of $0.02^{\circ}$. In other words, performance on the ASYNC task corresponds very closely to what one would predict on the basis of the eye movement hypothesis. It is worth noting that despite being above chance, performance is fairly low, as compared with earlier reports (Usher \& Donnelly, 1998). One possible reason for this is simply that results are reported for nonexperts, with only limited experience of psychophysical experimentation. The author was able to produce higher levels of performance under both conditions that were more in line with the levels reported by Usher and Donnelly.

\section{EXPERIMENT 3 Effects of Eye Movement Amplitude}

Although the results of Experiment 2 appear compelling, there are potential criticisms. It is not clear what effect viewing distance may have on issues such as the image jitter inherent in all CRT displays. It is also true that grid size covaried with viewing distance and that the size of the grid might be a contributing factor to the effect measured. Ultimately, the best means of assessing the role of eye movements is to measure them. In Experiment 3 , this was done directly by measuring eye location during performance of the task.

\section{Method}

The observers were once again shown a grid of elements in which alternate rows (or columns) appeared for a brief period, followed by the neighboring rows (or columns; ASYNC condition; see Figures $1 \mathrm{~A}$ and $1 \mathrm{~B}$ ) - in general accord with the temporal offset experiments of various previous studies (Lee \& Blake, 2001; Leonards et al., 1996; Parton et al., 2001; Usher \& Donnelly, 1998). In this case, however, the observers' eye and head movements were recorded using a binocular SMI EyeLink Series I eye tracker. This eye tracker uses video technology to sample eye and head location every $4 \mathrm{msec}$. The manufacturer reports it as having a spatial resolution of $0.005^{\circ}-$ that is, better than 20 arc sec. Gaze accuracy is considerably worse $\left(0.5^{\circ}\right.$ to $\left.1.0^{\circ}\right)$ but is not relevant to the measurements required in this study, which are concerned only with relative image motion over a very brief period. Calibration was based on a 9-point test grid. The $8 \times 8$ grid in Experiment $1 \mathrm{~A}$ was used, and the stimulus was viewed from $1,570 \mathrm{~mm}$, so that a single pixel subtended $0.01^{\circ} \times$ $0.01^{\circ}$, as in Experiment 1B. The image was generated by a custombuilt $2.4 \mathrm{GHz}$ PC using SMI display software running at a frame rate of $75 \mathrm{~Hz}$. The same Sony Trinitron monitor was used as that in the previous experiments. Image contrast was again set to $20 \%$. Eight subjects took part in this experiment, including 3 new, experienced psychophysical observers, the author, and 4 of the subjects used in the previous experiment.

\section{Results}

In order to estimate the relation between subject response and retinal image motion, the amplitude of such motion during image presentation had to be estimated. The eye tracker provides a measure of image motion across the retina that incorporates both head and eye movement. During stimulus presentation (duration, $13.3 \mathrm{msec}$ ), the eye tracker provided three or four measurements (one 
every $4 \mathrm{msec}$ ). The arithmetic mean of these measurements was used to give an estimate of the average image frame presentation location. The difference in the averages for the two image frames was taken as an estimate of the difference in presentation location of the two image frames. Finally, this value was averaged between the two eyes. This number was then binned into intervals of $0.01^{\circ}$ ranging from $0.00^{\circ}$ to $0.10^{\circ}$. The average proportion of trials falling within each bin can be seen in Figure 4A. As can be seen in the figure, the majority of eye movements $(55 \%)$ fell within the range of $0.02^{\circ}-0.05^{\circ}$. This proportion increased to $78 \%$ in the range of $0.01^{\circ}-0.06^{\circ}$ and to over $95 \%$ in the range of $0.00^{\circ}-0.10^{\circ}$. Trials excluded from the analysis included saccades $(4 \%$ of the trials on average), as well as other rapid head/eye movements. On the basis of the distributions presented in Figure $4 \mathrm{~A}$, an expected value of eye displacement can be calculated across all the trials of $0.10^{\circ}$ movement or less. This produces a figure of $0.036^{\circ}$. This figure is in close agreement with that predicted from the results in Experiment 1A, in which high levels of performance in a spatial offset task were reached only for offsets of around $0.03^{\circ}$ or $0.04^{\circ}$.

The overall performance of our subjects appear in Figure 4B. The bar chart presents the average value of $d^{\prime}$ for the 8 subjects over each interval of measured image shift. The figure reveals a clear upward trend in sensitivity to temporal offset as the amplitude of retinal image motion increases to $0.05^{\circ}$. Beyond $0.06^{\circ}$, sensitivity drops, becoming indiscernible from performance in the $0.00^{\circ}-0.01^{\circ}$

A

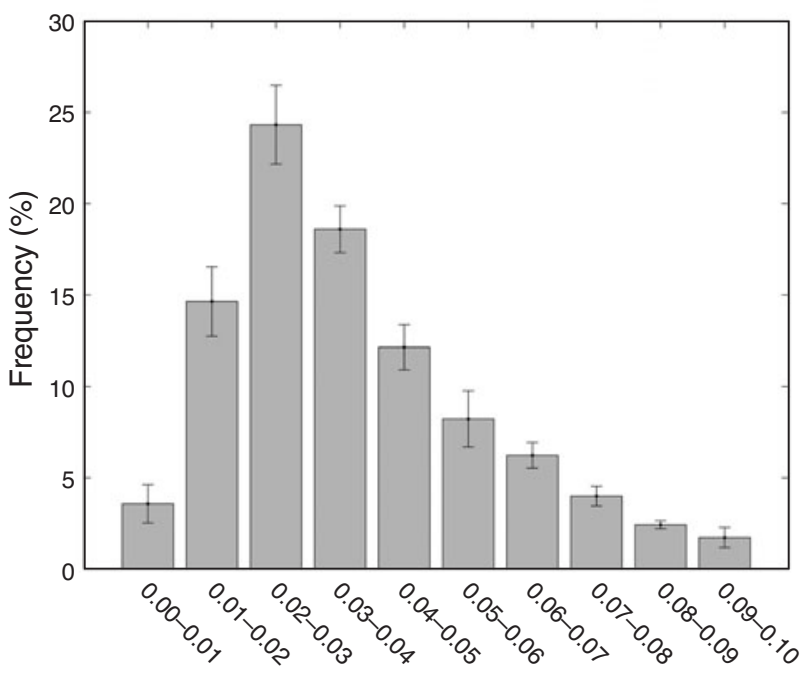

Retinal image displacement (deg) range. On the basis of these results, it appears that there is a limit to the benefit afforded by eye and head movements. Large eye shifts may start to enter the saccadic range and be subject to the effects of saccadic suppression and image blur. Despite this fact, the great majority of trials fall within the $0.01^{\circ}-0.06^{\circ}$ range. Within this range of image motion, performance remains significantly above chance.

The overall change in $d^{\prime}$ corresponds to a rise in performance from near chance at $55 \%$ for the range of $0.00^{\circ}-$ $0.01^{\circ}$, up to $80 \%$ in the range of $0.04^{\circ}-0.05^{\circ}$. Performance in the range of $0.00^{\circ}-0.01^{\circ}$ was, in fact, not significantly different from chance $[t(7)=1.123$, n.s. $]$. In other words, for very small eye movements, the subjects were choosing the orientation of the dot elements at or close to chance. The fact that the majority of image motion amplitudes fall in the useful range ensured that the subjects were performing well overall. Overall performance $\left(d^{\prime}=\right.$ 1.06) was significantly above chance $[F(1,7)=29.0$, $\left.M S_{\mathrm{e}}=0.91, p<.01\right]$. This level of performance was better than that in the previous experiment and was closer to the levels reported by Usher and Donnelly (1998).

\section{GENERAL DISCUSSION}

The purpose of this article has been to investigate whether retinal image motion can affect performance in the perceptual grouping of temporally offset stimuli. In so doing, the work has sought to offer a space-based explanation for reports that such grouping is affected by

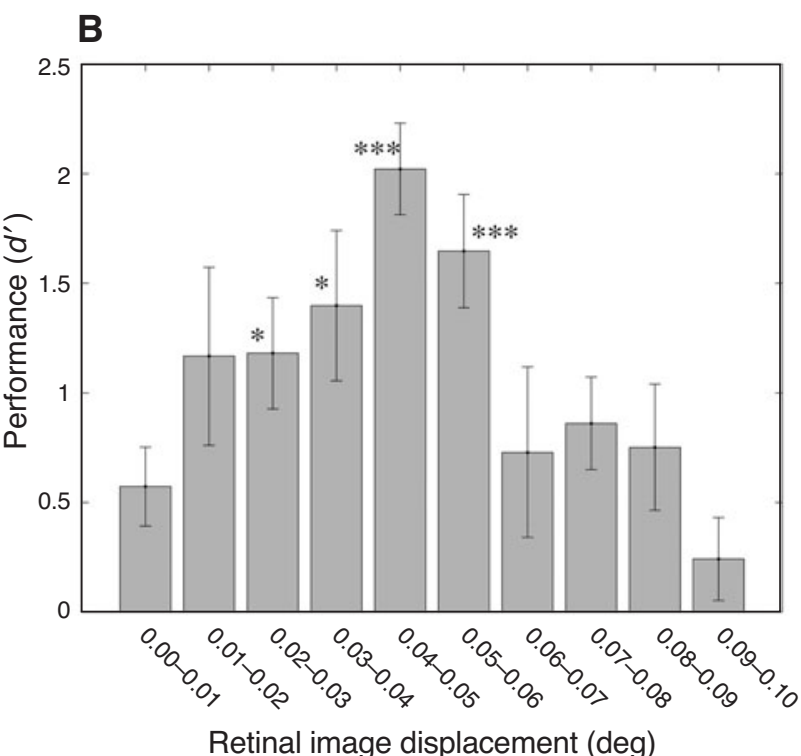

Figure 4. Results from Experiment 3, in which head and eye movements were recorded. (A) Eye movements were binned into intervals of $0.02^{\circ}$. The correspondence between the pattern of temporal offset and subject response was measured using $d^{\prime}$, and the graph presents averages across the 7 subjects. (B) Total number of eye movements recorded over each interval, revealing that the majority of eye movements were on the order of $0.01^{\circ}-0.05^{\circ}$, with an average of $0.036^{\circ}$. Error bars represent the standard error of the mean across subjects. Significant differences in performance between the lowest interval $\left(0.00^{\circ}-0.01^{\circ}\right)$ and the other intervals are indicated as follows: *p<.05, **p<.01, ***p<.005. 
brief temporal delays. The work provides strong evidence that eye and head movements do indeed affect performance in a classical row/column organization task and that they can, therefore, account for sensitivity to temporal asynchrony in tasks using grids of elements (Lee \& Blake, 2001; Leonards et al., 1996; Parton et al., 2001; Usher \& Donnelly, 1998).

As was described in the introduction, many reasons have been advanced as to why the results published in support of the temporal-binding hypothesis should be questioned. However, most of this work has focused on the Gabor fields used by Lee and Blake (2001) and others. The stimulus tested here is somewhat different, and it is therefore not clear what role each of the mechanisms described by these authors will play in the sensitivity measured here. In fact, this work demonstrates that earlier reports of sensitivity to temporal offsets of grids of dots may be explained in terms of eye and head movements alone. For other grid-based stimuli, eye movements are likely to be an important contributing factor (see Dakin \& Bex, 2002).

Beyond the rather limited range of grids of elements, it is unlikely that perceptual grouping tasks of almost any other stimuli would be affected by the miniscule spatial offsets generated by fixational image motion. It is interesting, therefore, that many attempts to influence perceptual grouping in other than grid-like patterns have thus far been unsuccessful (Fahle \& Koch, 1995; Keele, Cohen, Ivry, Liotti, \& Yee, 1988). As was described in the introduction, where exceptions to this rule have arisen, new studies have followed describing how the effects can be explained by such factors as onset effects, apparent motion artifacts, and so on (see Farid, 2002, for a review). This work has demonstrated that even given good fixation using a chinrest, retinal image motion caused by fixational eye and head movements can and does transform temporal offsets into apparent spatial ones.

So where does all of this evidence leave the temporalbinding hypothesis? It may be the case that one cannot tap into the temporal-binding mechanism in the manner attempted in the studies described here (Fahle \& Koch, 1995; Leonards et al., 1996) and, hence, that it is still a valid hypothesis. However, on the basis of the data reported here and in numerous earlier studies, it is safe to say that no compelling behavioral evidence to support the theory has thus far been produced.

If behavioral evidence for a temporal-binding mechanism does not exist, how might the binding problem otherwise be solved? One promising alternative to temporal binding is that we avoid the binding problem altogether, by not having multiple objects processed simultaneously but, instead, having attention switched from one object to the next (Desimone, Miller, Chelazzi, \& Lueschow, 1994; O’Regan, 1992; Reynolds \& Desimone, 1999; Treisman, 1998; Wolfe, 1994). Recent results from EEG studies have, however, revealed evidence that multiple objects can be processed in parallel right up to the highest levels of object representation (Rousselet, Fabre-Thorpe,
$\&$ Thorpe, 2002), suggesting that attention switching is not the whole story. Within the human ventral stream (long associated with object recognition), there is considerable evidence that local visual features are combined over several processing stages and that this may serve to avoid confusion of object attributes across an entire scene (Mel \& Fiser, 2000; Riesenhuber \& Poggio, 1999; Wallis \& Rolls, 1997). Single-cell recording work has further bolstered these types of models by demonstrating how ventral stream neurons can effectively filter out nonattended objects within their receptive field, in both the occipital (Chelazzi, Miller, Duncan, \& Desimone, 2001; Motter, 1993) and the temporal (Chelazzi, Duncan, Miller, \& Desimone, 1998; Rolls, Aggelopoulos, \& Zheng, 2003) cortices. Although this may offer a solution to binding within the ventral stream, it still leaves open the question of how other forms of information, such as location, motion, and a multitude of nonvisual representations, are bound. It is at this level that attention almost certainly plays a role. Attention has long been associated with the parietal lobe, and its role in binding is consistent with the fact that lesions of this lobe lead to numerous, classical object attribute integration problems (Farah, 1990). A multifaceted solution to the binding problem, of the type described here, is in fact entirely consistent with the latest neuropsychological and neurophysiological evidence (Robertson, 2003).

\section{REFERENCES}

Adelson, E. H., \& FARID, H. (1999). Filtering reveals form in temporally structured displays. Science, 286, 2231a.

AdLeR, F. H., \& Fliegelman, F. (1934). Influence of fixation on visual acuity. Archives of Ophthalmology, 12, 475-483.

BEAUDOT, W. H. (2002). Role of onset asynchrony in contour integration. Vision Research, 42, 1-9.

Ben-Av, M., \& SAGI, D. (1995). Perceptual grouping by similarity and proximity: Experimental results can be predicted by intensity autocorrelations. Vision Research, 35, 853-866.

Bolger, C., Bojanic, S., Sheahan, N., Coakley, D., \& Malone, J. (1999). Dominant frequency content of ocular microtremor from normal subjects. Vision Research, 39, 1911-1915.

Carpenter, R. H. S. (1988). Movements of the eyes. London: Pion.

Chelazzi, L., Duncan, J., Miller, E., \& Desimone, R. (1998). Responses of neurons in inferior temporal cortex during memory-guided visual search. Journal of Neurophysiology, 80, 2918-2940.

Chelazzi, L., Miller, E., Duncan, J., \& Desimone, R. (2001). Responses of neurons in macaque area V4 during memory-guided visual search. Cerebral Cortex, 11, 761-772.

CoAKley, D. (1983). Minute eye movement and brain stem function. Boca Raton, FL: CRC Press.

DAKIN, S. C., \& BEX, P. J. (2002). Role of synchrony in contour binding: Some transient doubts sustained. Journal of the Optical Society of America A, 19, 678-686.

Desimone, R., Miller, E., Chelazzi, L., \& Lueschow, A. (1994). Multiple memory systems in visual cortex. In M. S. Gazzaniga (Ed.), Cognitive neurosciences (pp. 475-486). Cambridge, MA: MIT Press.

FAHLE, M. (1993). Figure-ground discrimination from temporal information. Proceedings of the Royal Society of London: Series B, 254, 199-203.

FaHLe, M., \& КосH, C. (1995). Spatial displacement, but not temporal asynchrony, destroys figural binding. Vision Research, 35, 491-494.

FARAH, M. (1990). Visual agnosia: Disorders of object recognition and what they can tell us about normal vision. Cambridge, MA: MIT Press. 
FARID, H. (2002). Temporal synchrony in perceptual grouping: A critique. Trends in Cognitive Sciences, 6, 284-288.

FARID, H., \& ADELSON, E. (2001). Synchrony does not promote grouping in temporally structured displays. Nature Neuroscience, 4, 875876.

Felleman, D. J., \& Van Essen, D. C. (1991). Distributed hierarchical processing in the primate cerebral cortex. Cerebral Cortex, 1, 1-47.

Ferman, L., Collewijn, H., Jansen, T. C., \& Van den Berg, A. V. (1987). Human gaze stability in the horizontal, vertical and torsional direction during voluntary head movements, evaluated with a threedimensional scleral induction coil technique. Vision Research, 27, 811-828

Green, D. M., \& Swets, J. A. (1974). Signal detection theory and psychophysics. Huntington, NY: Krieger.

KANDIL, F., \& FAHLE, M. (2001). Purely temporal figure-ground segregation. European Journal of Neuroscience, 13, 2004-2008.

Keele, S., Cohen, A., Ivry, R., Liotti, M., \& Yee, P. (1988). Tests of a temporal theory of attentional binding. Journal of Experimental Psychology: Human Perception \& Performance, 14, 444-452.

KuHN, M. (2002). Optical time-domain eavesdropping risks of CRT displays. In Proceedings of the IEEE Symposium on Security and Privacy (pp. 3-18). Los Alamitos, CA: IEEE Computer Society Press.

LeE, S.-H., \& BlaKe, R. (1999a). Filtering reveals form in temporally structured displays: Response. Science, 286, 2231a.

LEE, S.-H., \& BLAKE, R. (1999b). Visual form created solely from temporal structure. Science, 284, 1165-1168.

LEE, S.-H., \& BLAKE, R. (2001). Neural synergy in visual grouping: When good continuation meets common fate. Vision Research, $\mathbf{4 1}$, 2057-2064.

LeOnards, U., Singer, W., \& Fahle, M. (1996). The influence of temporal phase differences on texture segmentation. Vision Research, $\mathbf{3 6}_{2}$ 2689-2697.

MALSBURG, C. VON DER (1995). Binding in models of perception and brain function. Current Opinion in Neurobiology, 5, 520-526.

MeL, B. W., \& FisER, J. (2000). Minimizing binding errors using learned conjunctive features. Neural Computation, 12, 731-762.

Mon-Williams, M., \& WanN, J. P. (1996). An illusion that avoids focus. Proceedings of the Royal Society of London: Series B, 263, 573-578.

Morgan, M., \& Castet, E. (2001). High temporal frequency synchrony is insufficient for perceptual grouping. Proceedings of the Royal Society of London: Series B, 269, 513-516.

Motter, B. (1993). Focal attention produces spatially selective processing in visual cortical areas V1, V2, and V4 in the presence of competing stimuli. Journal of Neurophysiology, 70, 909-919.

Murakami, I., \& CaVANaGH, P. (1998). A jitter after-effect reveals motion-based stabilization of vision. Nature, 395, 798-801.
O'Regan, J. (1992). Solving the "real" mysteries of visual perception: The world as an outside memory. Canadian Journal of Psychology, 46, 461-488.

PARTON, A., Donnelly, N., \& Usher, M. (2001). The effects of temporal synchrony on the perceived organization of elements in spatially symmetric and asymmetric grids. Visual Cognition, 8, 637-654.

RatLiff, F., \& RigGS, L. (1950). Involuntary motions of the eye during monocular fixation. Journal of Experimental Psychology, 40, 687701.

Reynolds, J. H., \& Desimone, R. (1999). The role of neural mechanisms of attention in solving the binding problem. Neuron, 24, 19-29.

Riesenhuber, M., \& Poggio, T. (1999). Are cortical models bound by the "binding problem"? Neuron, 24, 87-93.

Robertson, L. (2003). Binding, spatial attention and perceptual awareness. Nature Reviews Neuroscience, 4, 93-102.

Rolls, E. T., Aggelopoulos, N. C., \& Zheng, F. (2003). The receptive fields of inferior temporal cortex neurons in natural scenes. Journal of Neuroscience, 23, 339-348.

Rousselet, G. A., Fabre-Thorpe, M., \& Thorpe, S. J. (2002). Parallel processing in high-level categorization of natural images. Nature Neuroscience, 5, 629-630.

SHERR, S. (1993). Electronic displays. New York: Wiley.

Singer, W., \& GraY, C. (1995). Visual feature integration and the temporal correlation hypothesis. Annual Review of Neuroscience, $\mathbf{1 8}$, 555-586.

ToveE, M. J., \& Rolls, E. T. (1992). Oscillatory activity is not evident in the primate temporal visual cortex with static stimuli. NeuroReport, 3, 369-372.

Treisman, A. (1998). Feature binding, attention and object perception. Philosophical Transactions of the Royal Society of London: Series B, 353, $1295-1306$

Ungerleider, L., \& HaXby, J. (1994). "What" and "where" in the human brain. Current Opinion in Neurobiology, 4, 157-165.

UsHer, M., \& DonNELLY, N. (1998). Visual synchrony affects binding and segmentation in perception. Nature, 394, 179-182.

Wallis, G., \& Rolls, E. (1997). A model of invariant object recognition in the visual system. Progress in Neurobiology, 51, 167-194.

Wertheimer, M. (1923). Untersuchungen zur Lehre von der Gestalt. Psychologische Forschung, 4, 301-350.

Westheimer, G., \& McKee, S. (1977). Perception of temporal order in adjacent visual stimuli. Vision Research, 17, 887-892.

WOLFE, J. (1994). Visual search in continuous, naturalistic stimuli. Vision Research, 34, 1187-1195.

ZeKI, S., Watson, J. D. G., LueCK, C. J., Friston, K. J., Kennard, C., \& FracKowIAK, R. S. J. (1991). A direct demonstration of functional specialization in human visual cortex. Journal of Neuroscience, $\mathbf{1 1}$, 641-649.

\section{APPENDIX \\ Estimating the Maximum Spatial Offset Caused by Eye Tremor}

From Figure A1, panel A, we can see that the spatial offset between two consecutive frames, $\Psi$, depends on the average displacement of the eye during the two time periods from $t_{1}$ to $t_{2}$ and from $t_{2}$ to $t_{3}$. These average displacements can be calculated from the ratio of area of the regions $R_{1}$ and $R_{2}$ to their duration, as follows:

$$
\begin{aligned}
\Psi & =\frac{R_{1}}{t_{2}-t_{1}}-\frac{R_{2}}{t_{3}-t_{2}} \\
& =\frac{2}{T_{S}} \int_{t_{1}}^{t_{2}} A \sin \left(\frac{2 \pi t}{T_{E}}\right) d t-\frac{2}{T_{S}} \int_{t_{2}}^{t_{3}} A \sin \left(\frac{2 \pi t}{T_{E}}\right) d t \\
& =\frac{A T_{E}}{\pi T_{S}}\left[\cos \left(\frac{2 \pi t_{1}}{T_{E}}\right)-2 \cos \left(\frac{2 \pi t_{2}}{T_{E}}\right)+\cos \left(\frac{2 \pi t_{3}}{T_{E}}\right)\right] \\
& =\frac{A}{\alpha \pi}\{\cos (2 \pi \beta)-2 \cos [2 \pi(\alpha / 2+\beta)]+\cos [2 \pi(\alpha+\beta)]\},
\end{aligned}
$$

where $t_{3}-t_{1}=T_{S}=\alpha T_{E}$ and $t_{1}=\beta T_{E}$. 


\section{APPENDIX (Continued)}

Maxima and minima can be found for this function with respect to the ratios $\alpha$ and $\beta$ by equating the following partial differentials to zero:

$$
\begin{aligned}
& \frac{\partial \Psi}{\partial \alpha}=-\frac{A}{\alpha^{2}}\{\cos (2 \beta \pi)-2 \cos [(\alpha+2 \beta) \pi]+\cos [2(\alpha+\beta) \pi]\}+\frac{2 A}{\alpha}\{\sin [(\alpha+2 \beta) \pi]-\sin [2(\alpha+\beta) \pi]\} \\
& \frac{\partial \Psi}{\partial \beta}=-\frac{2 A}{\alpha}\{\sin (2 \beta \pi)-2 \sin [(\alpha+2 \beta) \pi]+\sin [2(\alpha+\beta) \pi]\} .
\end{aligned}
$$

Sample values from these equations are as follows:

\begin{tabular}{ccc}
$\Psi$ & $\alpha$ & $\beta$ \\
\hline $44 / \pi$ & $1 / 2$ & $1 / 4$ \\
$44 / \pi$ & 1 & 0 \\
0 & $1 / 2$ & 0 \\
$1.449 A$ & 0.742 & 0.129
\end{tabular}

The relationship of $\Psi$ to the ratio of $T_{E}$ to $T_{S}$ for the maximal, in-phase case $\left(t_{2}=T_{E} / 2\right)$ appears in Figure A1, panel B. Note that in general, for $T_{S}>T_{E}$, the average value of $\Psi$ one would expect over several trials drops as the ratio $T_{S}$ to $T_{E}$ increases. This is consistent with the findings reported by Usher and Donnelly (1998), in which sensitivity decreased as presentation duration $T_{S}$ increased from 32 to $160 \mathrm{msec}$. Current estimates put $T_{E}$ at between 10 and $25 \mathrm{msec}$ (Bolger et al., 1999). A spectral analysis of the image motion recorded in Experiment 3 (due to head and eye movement) revealed a peak at around $40 \mathrm{~Hz}$, with a much smaller but pronounced peak at $80 \mathrm{~Hz}$.

A

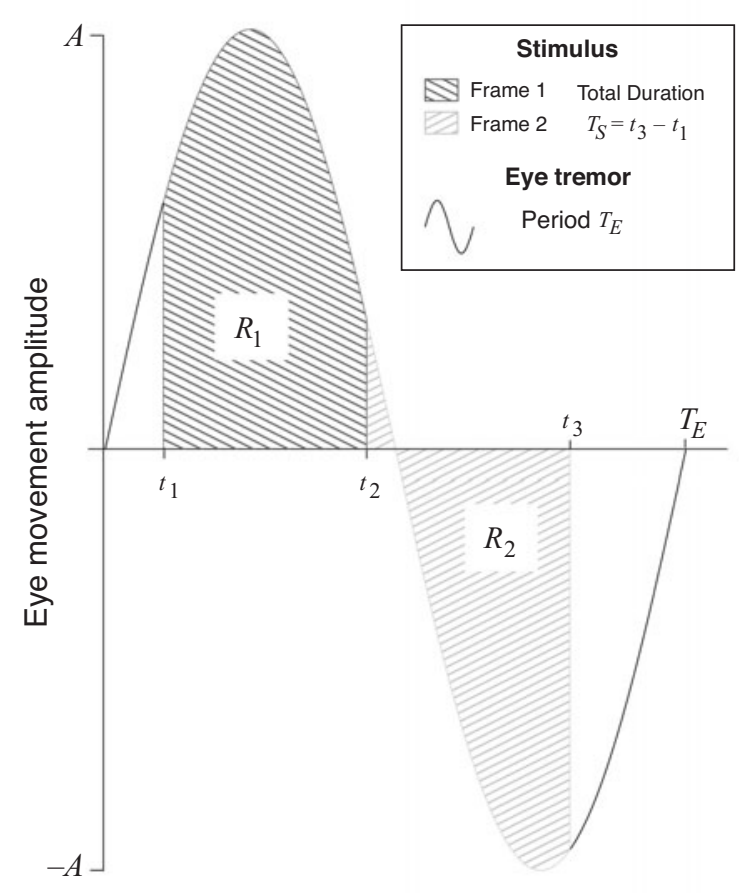

B

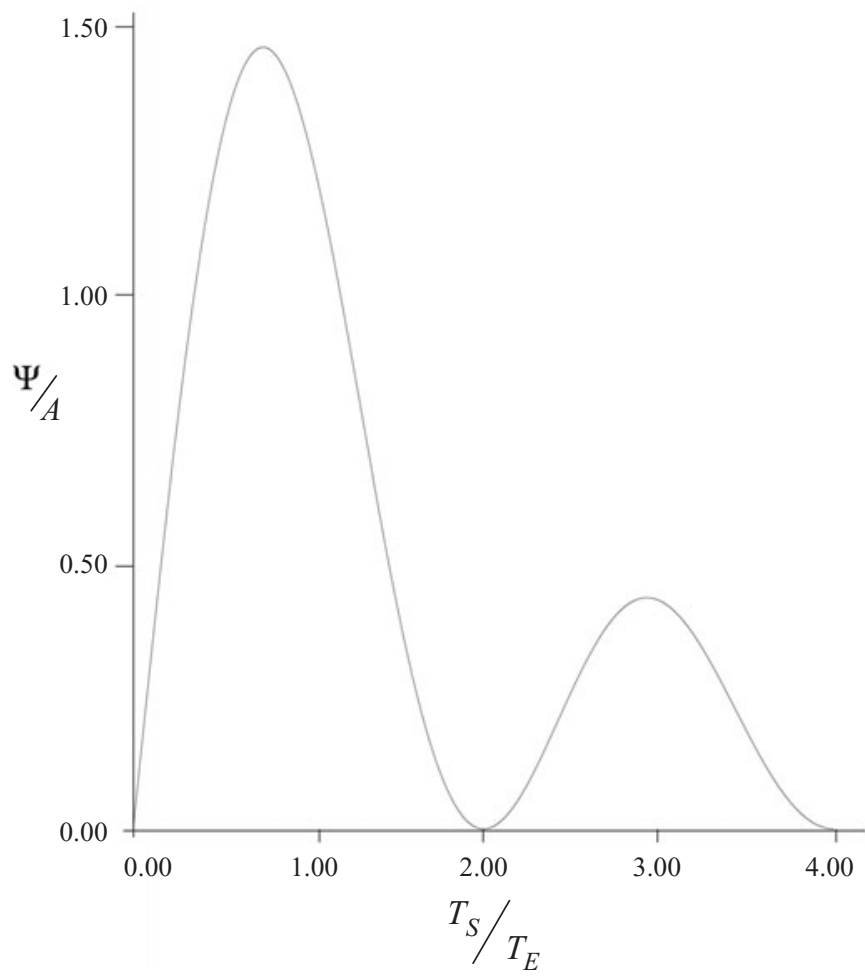

Figure A1. (A) A simplified model of eye tremor with amplitude $A$ and period $T_{E}$. Superimposed upon it are the periods during which the observer is exposed to the grid stimulus. Frame 1 appears in the interval from $t_{1}$ to $t_{2}$, and frame 2 in $t_{2}$ to $t_{3}$. Assuming that these two periods are of the same duration, this can be represented as a square-wave function of period $T_{s}$. An approximate value for the spatial offset of the two frames can be gauged from the difference in the average spatial location during the two intervals. (B) The maximum spatial offset $\psi$ achievable as a function of the ratio of the period of frame presentation $T_{S}$, to the period of eye movement oscillation $T_{E}$. The values are normalized to the amplitude of the eye movement $A$, and in order to maximize $\psi$, assume that the two functions are in phase. The graph reveals that spatial offset can vary from zero to nearly 1.5 times the amplitude of the oscillatory eye movement, depending on the relative duration of stimulus presentation and the frequency of oculomotor tremors. 